\title{
Overchoosing: A Mechanism of Tie-Formation in Social Networks
}

\author{
Tasuku Igarashi ${ }^{1}$ and Johan Koskinen ${ }^{2}$ \\ ${ }^{1}$ Graduate School of Education and Human Development, Nagoya University \\ ${ }^{2}$ Melbourne School of Psychological Sciences, The University of Melbourne
}

\begin{abstract}
Author Note
Tasuku Igarashi (D) https://orcid.org/0000-0001-9432-4425

Johan Koskinen (iD https://orcid.org/0000-0002-6860-325X

We have no conflicts of interest to disclose.

The research was supported by JSPS Fostering Joint International Research Program
\end{abstract} (KAKENHI; \#17KK0066).

We thank John Light for sharing his insights on the idea of the paper.

The research uses data from Add Health, a program project directed by Kathleen Mullan Harris and designed by J. Richard Udry, Peter S. Bearman, and Kathleen Mullan Harris at the University of North Carolina at Chapel Hill, and funded by grant P01-HD31921 from the Eunice Kennedy Shriver National Institute of Child Health and Human Development, with cooperative funding from 23 other federal agencies and foundations. Information on how to obtain the Add Health data files is available on the Add Health website (http://www.cpc.unc.edu/addhealth). No direct support was received from grant P01-HD31921 for this analysis. 
Correspondence concerning this article should be addressed to Tasuku Igarashi, Graduate School of Education and Human Development, Nagoya University, Furo-cho, Chikusa-ku, Nagoya, AICHI 464-8601 Japan. Email: igarashi.tasuku@d.mbox.nagoya-u.ac.jp 


\begin{abstract}
We introduce the concept of overchoosing as a fundamental mechanism of tie formation in directed social networks. The parameter represents a tendency for actors to send a lot of ties but receive few nominations back, something which implies the importance of modeling reciprocity violation as a basic tie-formation process. Analyzing large friendship network data $(N=1,575)$ by the stochastic actor-oriented models revealed that, under controlling for several endogenous tie formation processes, the parameter captured the formation of open triads and substantially improved the goodness of fit of the model to the data.
\end{abstract}

Keywords: social networks, reciprocity, outdegree popularity, stochastic actor-oriented models, Add Health 


\section{Overchoosing: A Mechanism of Tie-Formation in Social Networks}

Modeling social network dynamics is a key to understand the complexity of humans and society. This paper introduces the concept of overchoosing as a fundamental mechanism of tie formation in directed social networks. Overchoosing represents a tendency for actors to send a lot of ties but receive few nominations back, something which has been observed as an empirical phenomenon before (J. Light, personal communication, November 5, 2009). We define a statistic capturing the mechanism in terms of a negative interaction effect between outdegree popularity (star) and reciprocity:

$$
s_{i}(x)=\sum_{j} x_{i j} \sum_{h} x_{j h} \sum_{j} x_{i j} x_{j i}
$$

where $i, j$, and $h$ indicate actors, and $x_{i j}=1$ indicates the presence of a tie from actor $i$ to $j$ while $x_{i j}=0$ indicates the absence of the tie (see Figure 1).

\section{Figure 1}

Graphical representation of the process of overchoosing in a social network. Actor $j$ sending a lot of ties is likely to get few nominations back.

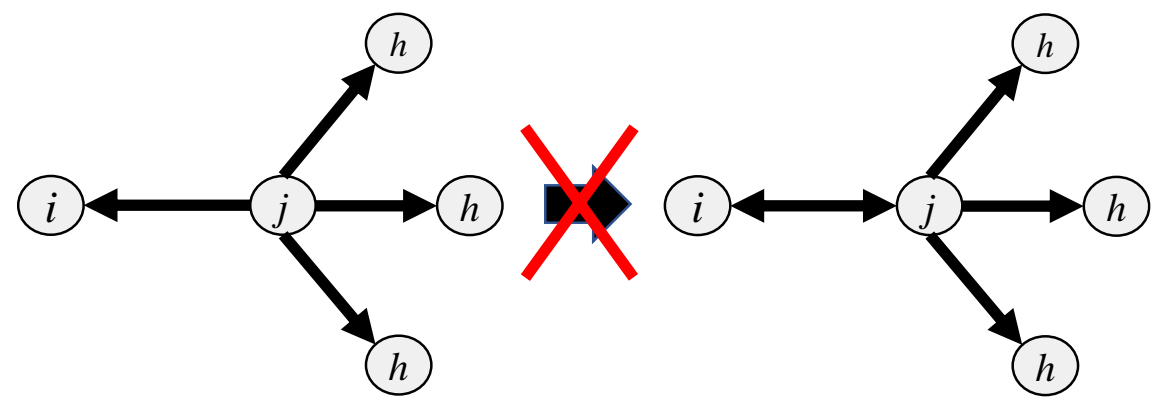

Like the proverb "throw enough mud at the wall and some of it will stick," the indiscriminate tie-formation strategy is supposed to be observed among some, probably a few, actors who are very active in nominating anyone accessible as friends or resource exchange 
partners in a given social setting (hereafter referred to as overchoosers). However, this would cause a backlash from some of the nominated actors (nominees) due to their lack of actual or perceived commitments and psychological closeness with the overchoosers (Gouldner, 1960; Rusbult, 1980; Thibaut \& Kelley, 1959). The mismatch between the overchoosers and the nominees would make the latter feel embarrassed to receive the ties and reluctant to send ties back to the former. A reciprocal tie-formation mechanism based on commitments at the dyadic level and reputations at the network level (Shiraki \& Igarashi, 2017) also explains the emergence of tie asymmetry — unless the overchoosers and the nominees have strong commitments, the nominees would be vigilant against negative reputations in the network through the involvement with the overchoosers who are poor at mindreading, or reckless, insensitive, and lacking in social skills to estimate appropriate psychological closeness with others.

Similar things also happen when nominations are personally made in questionnaires or interviews. Schematic representations of interpersonal relationships are generally reciprocal (De Soto \& Kuethe, 1959). However, it is not uncommon to find non-reciprocal ties in friendship networks because of the discrepancy of the friendship evaluation between actors (Carley \& Krackhardt, 1996). Overchoosers nominate a lot of others as friends, but many of them do not regard the overchoosers as friends.

Taking these into consideration, most of the nominations from the overchoosers would be one-sided (i.e. overchoosing), resulting in a low likelihood of reciprocal tie-formation. Although actors using the indiscriminate tie-formation strategy would not be the majority in social networks, their behavior could have a significant impact on the whole network dynamics, because of the large number of actors that are involved in the process as nominees. This degreerelated mechanism explicates not only the violation of reciprocity as a fundamental process of 
social network formation, but also the rare occurrence of open triangle including reciprocal tie(s) as its component.

The avoidance of overchoosers could also occur irrespective of whom they send ties to. In this case, the salience of overchoosing itself is a cause of the avoidance-overchoosers claim that he or she is their friends, but the target does not know who they are and then might gossip about it with others. Consequently, all actors in a given network could avoid forming ties with the outcasts due to their unpleasant impressions shared in the social environment. These patterns are simply formulated as the association between outdegrees and indegrees, or a negative interaction effect of outdegree and popularity (i.e. outdegree popularity), as reciprocation would not be incorporated as the core mediating mechanism here.

The two forms of overchoosing, one is reciprocity-based while the other is not, can be understood by the concepts of dyadic reciprocity and generalized reciprocity (Kenny \& La Voie, 1982; Kenny \& Nasby, 1980). Under the assumption of dyad interdependency, the two types of reciprocity have been investigated in a framework of the Social Relations Model (Kenny \& La Voie, 1984) that resolves the building blocks of interpersonal impressions into actor, target, and relationship effects based on round-robin research designs. Dyadic reciprocity means that if actor A likes actor B, actor B also likes actor A. Generalized reciprocity means that actor A who has an overall liking tendency toward others in a given social setting is also liked by others in general. In other words, the origin of dyadic reciprocity is attributed to the uniqueness of relationships among specific actors, whereas that of generalized reciprocity is attributed to individual-level factors that help the actor to achieve popularity among others in general. Dyadic reciprocity is found commonly in the measures of liking and self-disclosure especially among long-term acquaintances, whereas generalized reciprocity is not observed consistently (Kenny, 
1994; Miller \& Kenny, 1986). The findings imply that the reciprocity-based overchoosing effect (outdegree popularity $\times$ reciprocity) is more endogenous than the individual-based one (outdegree popularity) in social networks, and thus perhaps more robust to settle the questions of the "unobserved heterogeneity" in degree-related characteristics among actors.

The current paper analyzes large friendship networks to examine whether modeling the reciprocity-based overchoosing gives a comprehensive understanding of tie-formation dynamics in terms of reciprocity violation. Several research (Ball \& Newman, 2013; Cheng et al., 2011; Jiang et al., 2015) has also investigated the unreciprocated nature of social ties, but these models do not include other substantial tie-formation processes such as triadic closure. In this study, the Stochastic Actor-Oriented Models (SAOM; Snijders, 2001) were employed to examine the generative network process after controlling for several endogenous factors of dynamic tie formation.

Saturated friendship network data from the National Longitudinal Study of Adolescent to Adult Health (Add Health; Harris et al., 2009) was used to examine the overchoosing mechanism. The data was processed based on an open-source script (Bishop, 2015) for R and analyzed by RSiena (Ripley et al., 2019). Two large schools $\left(N \mathrm{~s}=562\right.$ and 1,013; $M_{\text {age }}=15.6$ years old) were selected from the data due to low attrition rates (<20\%; Simpkins et al., 2013). Each actor was asked to nominate up to ten friends (five males and females, respectively) at each of the two waves.

In SAOM, Model 1 (baseline) included the structural effects of outdegree, reciprocity, GWESP [geometrically weighted edgewise shared partners] FF [forward-forward] (i.e. triadic closure), GWESP BB [backward-backward] (i.e. cyclic closure), indegree (square-root) and outdegree popularity, outdegree activity, zero and low outdegree, and reciprocity $\times$ GWESP FF 
(Block, 2015). Model 2 was identical with Model 1 except that it included the overchoosing effect (outdegree popularity $\times$ reciprocity). As another form of reciprocity violation, the interaction effect of indegree popularity $\times$ reciprocity (i.e. underchoosing; actors receiving many ties are less likely to send all back) was also included (see Appendix for a sample RSiena script).

Figure 2 shows scatter plots of outdegrees and reciprocal degrees (blue)/indegrees (red) alongside of regression slopes, indicating that actors nominating greater number of others tended to have smaller number of reciprocal ties and smaller number of nominations than they made. Note that actors whose outdegrees were six or more did not receive full nominations back (i.e. no points were along the diagonals at $x \geq 6$ in the plots of reciprocal degrees).

Table 1 shows the results of parameter estimations for the two schools. In Model 1, the outdegree popularity effect was negative and significant. In Model 2, both of the outdegree popularity and the outdegree popularity $\times$ reciprocity effects were negative and significant. Figure 3 shows goodness-of-fit statistics for triad census (Lospinoso \& Snijders, 2019). In Model 1, the triadic properties of the simulated networks did not fit well to those of the observed networks. However, the inclusion of the outdegree popularity $\times$ reciprocity effect in Model 2 substantially improved the fit of the model to the data, especially for open triads (021D and 021C; no mutual tie and two asymmetric ties in downward and cyclic structure). 


\section{Figure 2}

Scatter plots of outdegrees and reciprocal degrees (blue) and indegrees (red). Black lines indicate diagonals (where the number of reciprocal degrees/indegrees and outdegrees are equivalent). Blue and red lines indicate regression slopes on reciprocal degrees and indegrees.

\section{School 1}
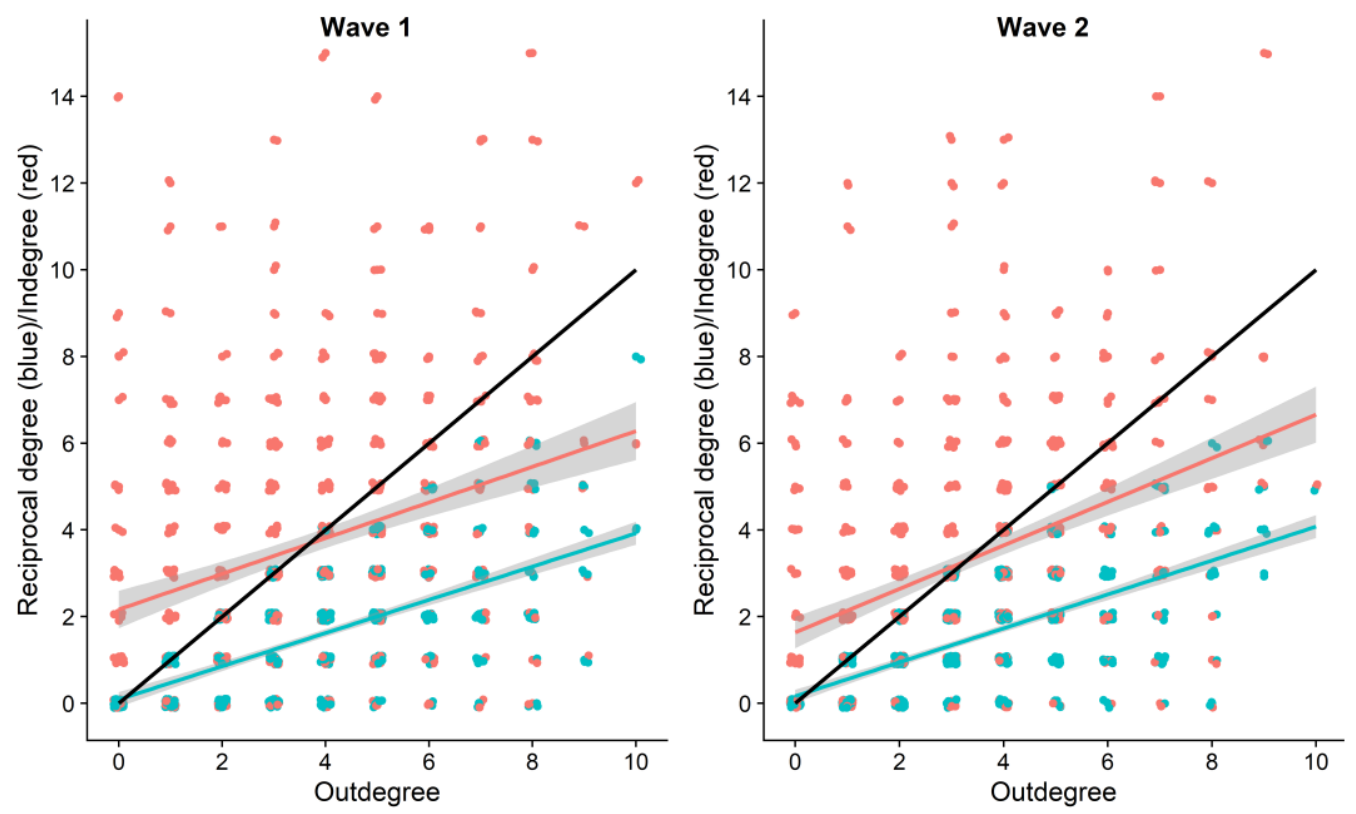

\section{School 2}
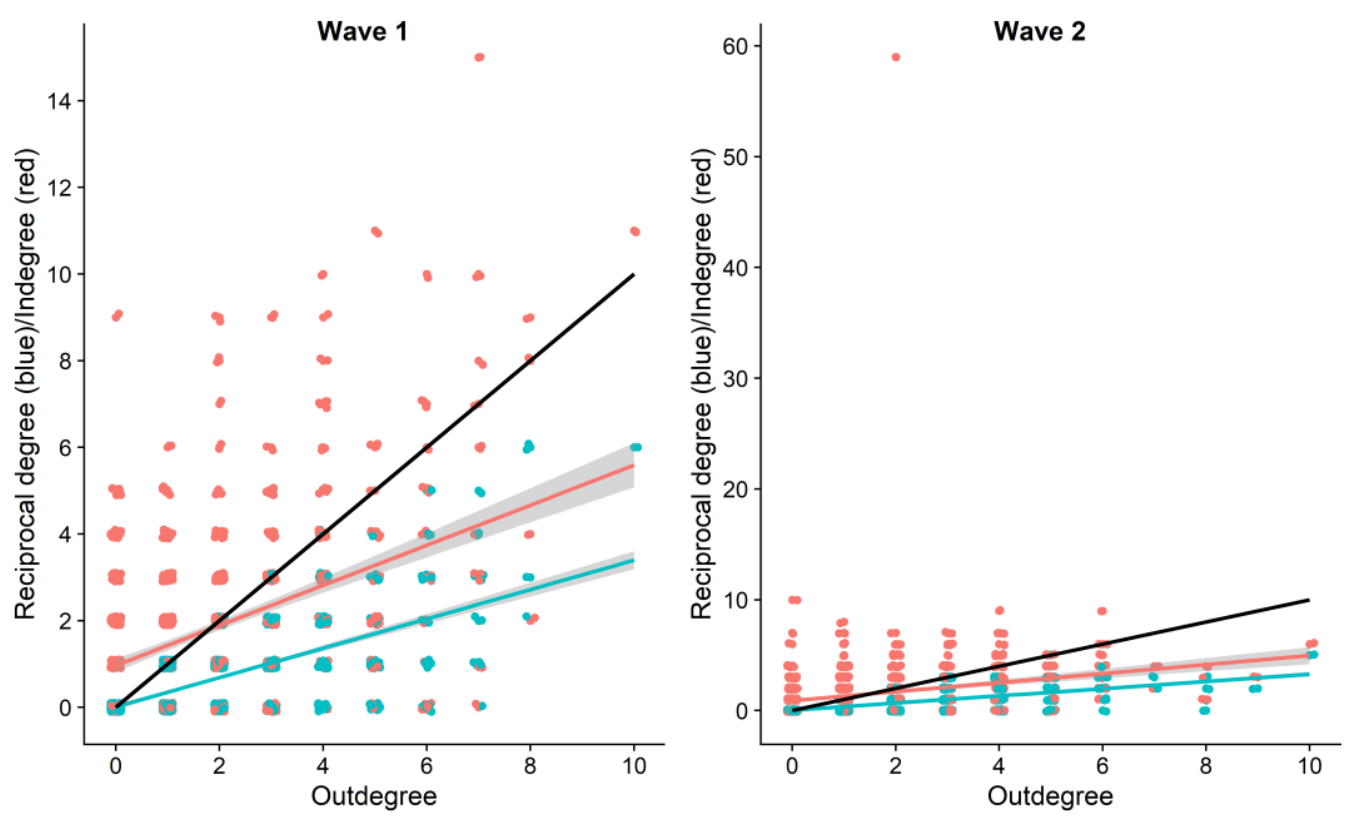


\section{Table 1}

Parameter estimation (conditional log-odds) in Stochastic Actor-Oriented Models.

\begin{tabular}{|c|c|c|c|c|c|c|c|c|}
\hline \multirow[b]{3}{*}{ Effect } & \multicolumn{4}{|c|}{ School $1(N=562)$} & \multicolumn{4}{|c|}{ School $2(N=1,013)$} \\
\hline & \multicolumn{2}{|c|}{ Model 1} & \multicolumn{2}{|c|}{ Model 2} & \multicolumn{2}{|c|}{ Model 1} & \multicolumn{2}{|c|}{ Model 2} \\
\hline & Estimate & (SE) & Estimate & $(\mathrm{SE})$ & Estimate & (SE) & Estimate & $(\mathrm{SE})$ \\
\hline Rate & 13.113 & $(0.691)$ & 12.768 & $(0.662)$ & 7.611 & $(0.462)$ & 7.065 & $(0.394)$ \\
\hline Outdegree (density) & $-3.221 * * *$ & $(0.186)$ & $-3.661 * * *$ & $(0.181)$ & $-3.775 * * *$ & $(0.429)$ & $-4.240 * * *$ & $(0.338)$ \\
\hline Reciprocity & $3.292 * * *$ & $(0.106)$ & $4.963 * * *$ & $(0.363)$ & $4.080 * * *$ & $(0.211)$ & $5.973 * * *$ & $(0.421)$ \\
\hline GWESP FF & $2.304 * * *$ & $(0.083)$ & $2.190 * * *$ & $(0.079)$ & $3.136 * * *$ & $(0.133)$ & $2.986 * * *$ & $(0.153)$ \\
\hline GWESP BB & $0.536 * * *$ & $(0.085)$ & $0.672 * * *$ & $(0.085)$ & $0.580 * *$ & $(0.211)$ & $1.004 * * *$ & $(0.174)$ \\
\hline Indegree popularity (square root) & $0.325 * * *$ & $(0.048)$ & $0.366 * * *$ & $(0.050)$ & $0.649 * * *$ & $(0.154)$ & $0.717 * * *$ & $(0.133)$ \\
\hline Outdegree popularity & $-0.184 * * *$ & $(0.027)$ & $-0.117 * * *$ & $(0.028)$ & $-0.361 * * *$ & $(0.086)$ & $-0.266 * * *$ & $(0.073)$ \\
\hline Outdegree activity & $-0.044 * *$ & $(0.015)$ & -0.025 & $(0.014)$ & $-0.090 *$ & $(0.040)$ & -0.055 & $(0.030)$ \\
\hline Zero outdegree & 0.215 & $(1.001)$ & 0.202 & $(0.926)$ & 1.879 & $(0.977)$ & $1.868 *$ & $(0.768)$ \\
\hline Low outdegree (1) & -0.238 & $(0.495)$ & -0.236 & $(0.460)$ & $-1.606 *$ & $(0.675)$ & $-1.649 * *$ & $(0.541)$ \\
\hline GWESP FF $\times$ Reciprocity & $-2.200 * * *$ & $(0.150)$ & $-1.794 * * *$ & $(0.193)$ & $-2.902 * * *$ & $(0.244)$ & $-2.256 * * *$ & $(0.385)$ \\
\hline Outdegree popularity $\times$ Reciprocity & & & $-0.348 * * *$ & $(0.090)$ & & & $-0.630 * * *$ & $(0.143)$ \\
\hline Indegree popularity $\times$ Reciprocity & & & -0.074 & $(0.041)$ & & & -0.081 & $(0.101)$ \\
\hline Overall maximum convergence ratio & 0.07 & & 0.09 & & 0.06 & & 0.09 & \\
\hline
\end{tabular}

Note. ${ }^{*} p<.05 ; * * p<.01 ; * * * p<.001$. 
Figure 3

Goodness-of-fit diagnostic plot with triad census distribution (Holland \& Leinhardt, 1970) as auxiliary statistic. Numbers indicate observed values of given triadic structure (connected by lines). Violin plots indicate simulated statistics. P-values indicate the results of the Mahalanobis distance-based model fitting tests ( $p>.05$ shows a reasonable fit).

\section{School 1}

Model 1

Goodness of Fit of TriadCensus

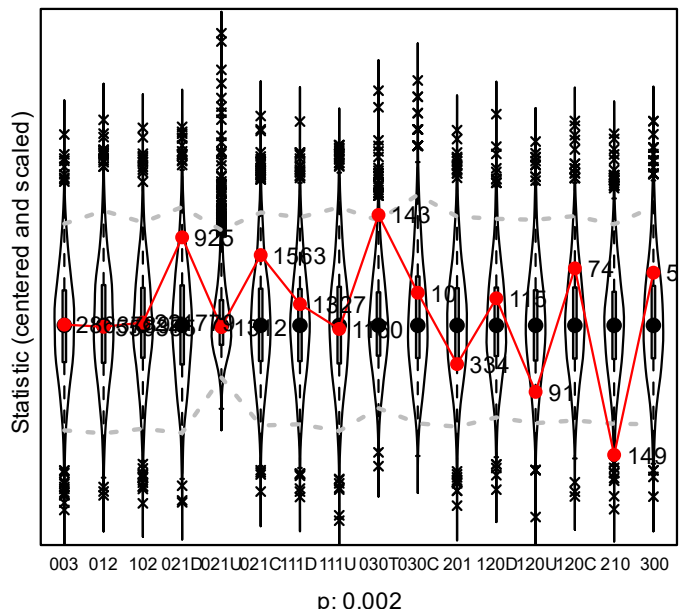

Model 2

Goodness of Fit of TriadCensus

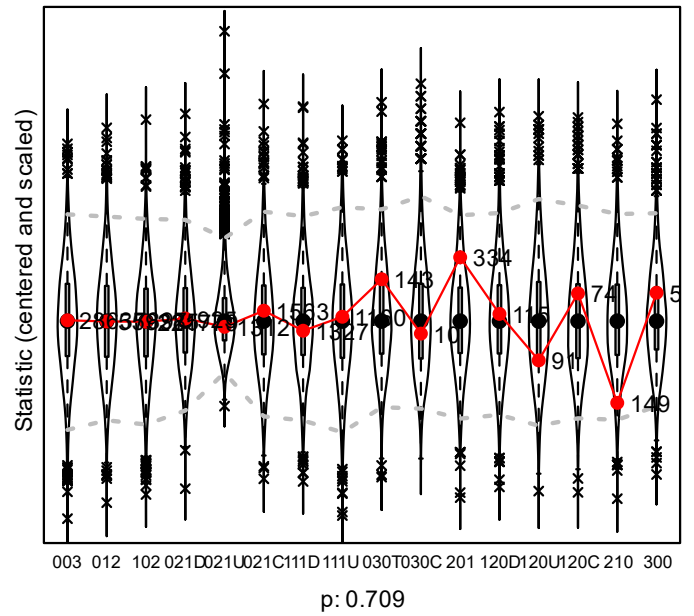

School 2

Model 1

Goodness of Fit of TriadCensus

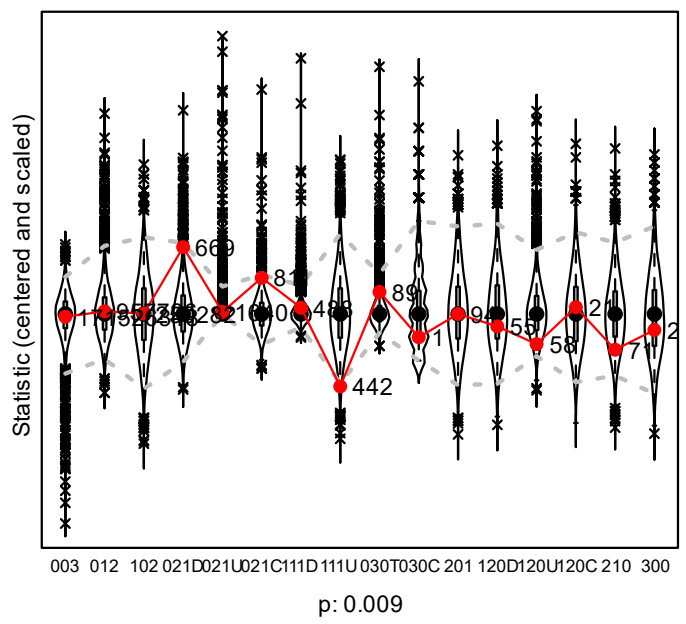

Model 2

Goodness of Fit of TriadCensus

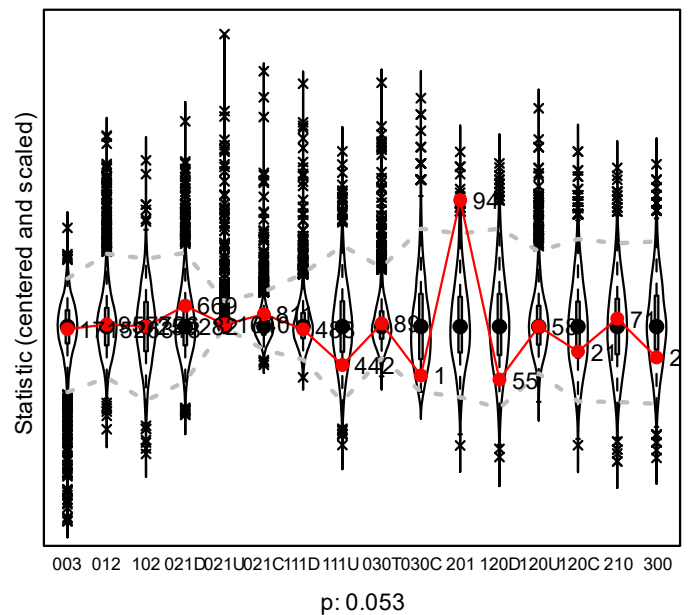


The findings show the importance of modeling reciprocity violation as a fundamental tieformation process in social networks, which provides a novel understanding of the generative process of open triangle in relation to degree-related distributions under controlling for endogenous triangle formation mechanisms. It is notable that the outdegree popularity effect was still significant in Model 2. The finding reinforces the idea that overchoosing is indeed a mechanism that is directly related to reciprocity, and not a general "pariah" effect.

The parameterization of reciprocity-based overchoosing is expected to guide researchers to select appropriate models that reasonably fit to actual data and can be easily extended to other frameworks of social network modeling, such as Exponential Random Graph Models and Relational Event Models. It is also of interest to model tie-formation processes in online social networks by the effect, in which overchoosing will be more evident than in offline social networks due to the open nature of social environments.

There is also a great deal of common ground between the lack of six or more reciprocal ties in the school friendship network data and the typical size of support clique (four to five) each individual holds to seek emotional supports in a single community (Dunbar, 2018; Sutcliffe et al., 2012). This gives a significant insight on the nature of overchoosing with respect to lack of an ability to gauge one's psychological closeness with others. Overchoosing may be represented as a categorical rather than continuous characteristic of some actors in a network who tend to overestimate their closeness with others out of the innermost social circle and receive few nominations back.

The idea of overchoosing encapsulates the discrepancy in psychological closeness among actors and poor social embeddedness of overchoosers as its consequence. This is more than mere social isolation and implies that the effect might be useful for the assessment of whether there are 
actors who become maladaptive through the process of seeking social connections but not being accepted by others, which could be a source of loneliness (Peplau \& Perlman, 1979). Modeling the parameter in correspondence with psychological characteristics would give further understanding of the dynamic construction of human minds and social environments. 


\section{References}

Ball, B., \& Newman, M. E. J. (2013). Friendship networks and social status. Network Science, 1(1), 1630. https://doi.org/10.1017/nws.2012.4

Bishop, M. M. (2015). netAddHealth: Network analysis using longitudinal study of adolescent health. In https://github.com/MichaelMBishop/netAddHealth

Block, P. (2015). Reciprocity, transitivity, and the mysterious three-cycle. Social Networks, 40, 163-173. https://doi.org/10.1016/j.socnet.2014.10.005

Carley, K. M., \& Krackhardt, D. (1996). Cognitive inconsistencies and non-symmetric friendship. Social Networks, 18(1), 1-27. https://doi.org/https://doi.org/10.1016/0378-8733(95)00252-9

Cheng, J., Romero, D. M., Meeder, B., \& Kleinberg, J. (2011). Predicting reciprocity in social networks. 2011 IEEE Third International Conference on Privacy, Security, Risk and Trust and 2011 IEEE Third International Conference on Social Computing, Boston, MA, USA.

De Soto, C. B., \& Kuethe, J. L. (1959). Subjective probabilities of interpersonal relationships. The Journal of Abnormal and Social Psychology, 59(2), 290-294. https://doi.org/10.1037/h0045430

Dunbar, R. I. M. (2018). The anatomy of friendship. Trends in Cognitive Sciences, 22(1), 32-51. https://doi.org/10.1016/j.tics.2017.10.004

Gouldner, A. W. (1960). The norm of reciprocity: A preliminary statement. American Sociological Review, 25(2), 161-178. https://doi.org/10.2307/2092623

Harris, K. M., Halpern, C. T., Whitsel, E., Hussey, J., Tabor, J., Entzel, P., \& Udry, J. R. (2009). The national longitudinal study of adolescent to adult health: Research design. http://www.cpc.unc.edu/projects/addhealth/design

Holland, P. W., \& Leinhardt, S. (1970). A method for detecting structure in sociometric data. American Journal of Sociology, 76(3), 492-513. https://doi.org/10.1086/224954 
Jiang, B., Zhang, Z.-L., \& Towsley, D. (2015). Reciprocity in social networks with capacity constraints. Proceedings of the 21th ACM SIGKDD International Conference on Knowledge Discovery and Data Mining, Sydney, NSW, Australia.

Kenny, D. A. (1994). Interpersonal perception: A Social Relations Analysis. New York: Guilford.

Kenny, D. A., \& La Voie, L. (1982). Reciprocity of interpersonal attraction: A confirmed hypothesis. Social Psychology Quarterly, 45(1), 54-58. https://doi.org/10.2307/3033675

Kenny, D. A., \& La Voie, L. (1984). The social relations model. Advances in experimental social psychology, 18, 142-182. https://doi.org/https://doi.org/10.1016/S0065-2601(08)60144-6

Kenny, D. A., \& Nasby, W. (1980). Splitting the reciprocity correlation. Journal of Personality and Social Psychology, 38(2), 249-256. https://doi.org/https://doi.org/10.1037/0022-3514.38.2.249

Lospinoso, J., \& Snijders, T. A. B. (2019). Goodness of fit for stochastic actor-oriented models. Methodological Innovations, 12(3), 1-18. https://doi.org/10.1177/2059799119884282

Miller, L. C., \& Kenny, D. A. (1986). Reciprocity of self-disclosure at the individual and dyadic levels: A social relations analysis. Journal of Personality and Social Psychology, 50(4), 713-719. https://doi.org/10.1037/0022-3514.50.4.713

Peplau, L. A., \& Perlman, D. (1979). Blueprint for a social psychological theory of loneliness. In M. Cook \& G. WIlson (Eds.), Love and attraction: An interpersonal conference (pp. 101-110). Pergamon Press.

Ripley, R. M., Snijders, T. A. B., Boda, Z., Vörös, A., \& Preciado, P. (2019). Manual for RSiena. https://www.stats.ox.ac.uk/ snijders/siena/RSiena_Manual.pdf

Rusbult, C. E. (1980). Commitment and satisfaction in romantic associations: A test of the investment model. Journal of Experimental Social Psychology, 16(2), 172-186. https://doi.org/10.1016/0022$\underline{1031(80) 90007-4}$

Shiraki, Y., \& Igarashi, T. (2017). We can't return evil for good: The comparison between direct and indirect reciprocity. Letters on Evolutionary Behavioral Science, 8(1), 4-7. https://doi.org/10.5178/lebs.2017.54 
Simpkins, S. D., Schaefer, D. R., Price, C. D., \& Vest, A. E. (2013). Adolescent friendships, BMI, and physical activity: Untangling selection and influence through longitudinal social network analysis. Journal of Research in Adolescence, 23(3), 537-549. https://doi.org/10.1111/j.15327795.2012.00836.x

Snijders, T. A. B. (2001). The statistical evaluation of social network dynamics. In M. P. Becker (Ed.), Sociological Methodology (pp. 361-395). Basil Blackwell.

Sutcliffe, A., Dunbar, R., Binder, J., \& Arrow, H. (2012). Relationships and the social brain: Integrating psychological and evolutionary perspectives. British Journal of Social Psychology, 103, 149-168. https://doi.org/10.1111/j.2044-8295.2011.02061.x

Thibaut, J. W., \& Kelley, H. H. (1959). The social psychology of groups. John Wiley. 


\section{Appendix}

A sample RSiena script for modeling overchoosing. The variable mydata is a Siena data object.

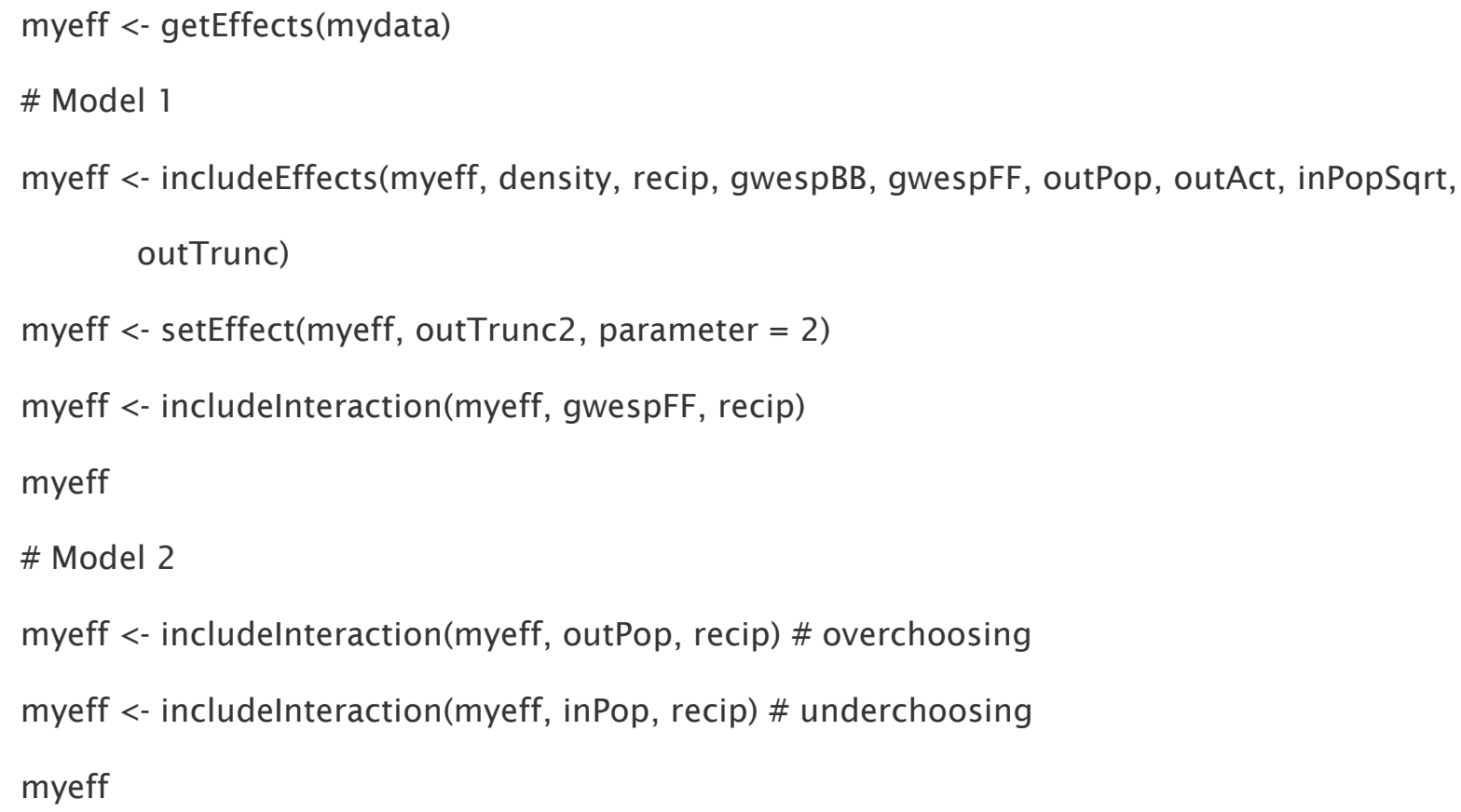

